\title{
High Precision Evaluation of the Irregular Coulomb Wave Functions
}

\author{
By A. J. Strecok and J. A. Gregory*
}

\begin{abstract}
This tutorial paper presents practical methods for accurately evaluating irregular Coulomb wave functions. Rational approximations to $G_{0}(\eta, \rho)$ and $G_{0}^{\prime}(\eta, \rho)$ are developed along line segments in the $(\eta, \rho)$ plane to provide useful initial values for the associated differential equation. These approximations, designed for IBM System 360 Fortran double precision, yield results to at least 13 significant decimal places.
\end{abstract}

1. Introduction. Considerable interest in Coulomb wave functions developed from physical problems involving collisions of charged particles. These functions $F_{L}(\eta, \rho)$ and $G_{L}(\eta, \rho)$, called the regular and irregular Coulomb wave functions, are two linearly independent solutions of the second-order differential equation

$$
d^{2} y / d \rho^{2}+\left[1-2 \eta / \rho-L(L+1) / \rho^{2}\right] y=0
$$

for real $\eta$, positive $\rho$, and nonnegative integer $L$.

The regular Coulomb wave functions are readily evaluated using well-known methods [3], [9]. A useful three-term recurrence relation technique for generating $F_{L}(\eta, \rho)$, working from higher $L$ values to lower ones, is discussed in detail by Gautschi [10], [11]. Unfortunately, because of the different asymptotic properties with respect to $L$ between the regular and irregular Coulomb wave functions, this technique cannot be safely applied to the irregular functions. A generation from lower to higher $L$ values using the recurrence relation is possible, but then the exact numerical values of two consecutive $G_{L}(\eta, \rho)$ functions are required.

Because irregular Coulomb wave functions are difficult to compute, current tables [2], [3], [8], [17], [19], [23] contain relatively few digits. As examples, taken from Luke [16], which indicate computational difficulties that can be encountered, consider the related equations

$$
\begin{aligned}
F_{L}(\eta, \rho)+ & i G_{L}(\eta, \rho) \\
& =\frac{(-1)^{L+1} \Gamma(L+1-i \eta) e^{\pi \eta / 2}(2 \rho)^{L+1} e^{-i \rho}}{|\Gamma(L+1-i \eta)|} \Psi(L+1-i \eta ; 2 L+2 ; 2 i \rho)
\end{aligned}
$$

and

$$
F_{L}(\eta, \rho)+i G_{L}(\eta, \rho)=\frac{\Gamma(L+1-i \eta) e^{\pi \eta / 2} i^{L+1}}{|\Gamma(L+1-i \eta)|} W_{i \eta, L+1 / 2}(2 i \rho)
$$

Received February 18, 1972.

AMS 1970 subject classifications. Primary 65D20, 65D25, 65D30.

Key words and phrases. Coulomb wave functions, differential equations, rational approximations.

* Work performed under the auspices of the U. S. Atomic Energy Commission at Argonne National Laboratory, Argonne, Illinois 60439.

Copyright @ 1972, American Mathematical Society 
where

$$
\Psi(a ; c ; z)=\int_{0}^{\infty} e^{-z t} t^{a-1}(1+t)^{c-a-1} d t / \Gamma(a), \quad R(a)>0, \quad R(z)>0,
$$

is the confluent hypergeometric function and $W_{k, m}(z)$ is the Whittaker confluent hypergeometric function. In fact, a method for computing irregular Coulomb wave functions using Whittaker functions was recently developed [5], and found to work fairly well for small parameters, but was subject to severe cancellations for larger ones [20]. Many well-known formulas are either too specialized, too complicated, or not sufficiently accurate to be immediately useful for the uninitiated user. Furthermore, errors in earlier works are noted in more recent literature [9], [13]. Some of these difficulties arise from attempts to represent $G_{L}(\eta, \rho)$ directly in terms of all three parameters simultaneously.

In this report, we develop accurate but relatively simple approximations for $G_{0}(\eta, \rho)$ and $G_{0}^{\prime}(\eta, \rho)$ along certain line segments in the positive region of the $(\eta, \rho)$ plane, eliminating the complications of three-dimensional representations. The coefficients for these approximation formulas are given in tables on the microfiche card attached to this issue.

2. Basic Relations. Some of the formulas on which this work is based were previously given by Abramowitz [3], Fröberg [9], and others. The most useful of these are summarized below.

$$
\begin{gathered}
\left(1+\eta^{2}\right)^{1 / 2} \cdot \Gamma_{1}=\left(\rho^{-1}+\eta\right) \cdot \Gamma_{0}-\Gamma_{0}^{\prime}, \\
L\left[(L+1)^{2}+\eta^{2}\right]^{1 / 2} \cdot \Gamma_{L+1} \\
=(2 L+1)[\eta+L(L+1) / \rho] \cdot \Gamma_{L}-(L+1)\left(L^{2}+\eta^{2}\right)^{1 / 2} \cdot \Gamma_{L-1},
\end{gathered}
$$

where $\Gamma_{L}$ can be taken as $F_{L}(\eta, \rho)$ or $G_{L}(\eta, \rho)$.

$$
\begin{aligned}
F_{L}+i G_{L}=\frac{e^{-\pi \eta} \rho^{L+1}}{(2 L+1) ! C_{L}(\eta)} \int_{0}^{\infty} & \left\{\left(1-\tanh ^{2} t\right)^{L+1} \exp [-i(\rho \tanh t-2 \eta t)]\right. \\
& \left.+i\left(1+t^{2}\right)^{L} \exp [-\rho t+2 \eta \arctan t]\right\} d t
\end{aligned}
$$

where

$$
\begin{aligned}
& C_{0}(\eta)=\left(2 \pi \eta\left(e^{2 \pi \eta}-1\right)^{-1}\right)^{1 / 2} \quad \text { and } \quad C_{L}(\eta)=\frac{\left(L^{2}+\eta^{2}\right)^{1 / 2}}{L(2 L+1)} C_{L-1}(\eta) \\
& \begin{array}{l}
F_{0}(\eta, \rho) \\
G_{0}(\eta, \rho)
\end{array} \pi^{1 / 2}(2 \eta)^{1 / 6}\left\{\begin{array}{l}
\operatorname{Ai}(x) \\
\operatorname{Bi}(x)
\end{array}\left[1+\frac{g_{1}}{\mu}+\frac{g_{2}}{\mu^{2}}+\cdots\right]\right. \\
& \left.+\underset{\mathrm{Bi}^{\prime}(x)}{\mathrm{Ai}^{\prime}(x)}\left[\frac{f_{1}}{\mu}+\frac{f_{2}}{\mu}+\cdots\right]\right\} \text {, } \\
& \begin{array}{l}
F_{0}^{\prime}(\eta, \rho) \\
G_{0}^{\prime}(\eta, \rho)
\end{array}-\pi^{1 / 2}(2 \eta)^{-1 / 6}\left\{\begin{array}{l}
\operatorname{Ai}(x) \\
\operatorname{Bi}(x)
\end{array}\left[\frac{g_{1}^{\prime}+x f_{1}}{\mu}+\frac{g_{2}^{\prime}+x f_{2}}{\mu^{2}}+\cdots\right]\right. \\
& \left.+\underset{\mathrm{Bi}^{\prime}(x)}{\mathbf{A i}^{\prime}(x)}\left[1+\frac{\left(g_{1}+f_{1}^{\prime}\right)}{\mu}+\frac{\left(g_{2}+f_{2}^{\prime}\right)}{\mu^{2}}+\cdots\right]\right\},
\end{aligned}
$$


where

$$
\begin{aligned}
f_{1} & =x^{2} / 5 \\
f_{2} & =\left(2 x^{3}+6\right) / 35, \\
f_{3} & =\left(84 x^{7}+1480 x^{4}+2320 x\right) / 63000, \\
g_{1} & =-x / 5 \\
g_{2} & =\left(7 x^{5}-30 x^{2}\right) / 350 \\
g_{3} & =\left(1056 x^{6}-1160 x^{3}-2240\right) / 63000, \\
x & =(2 \eta-\rho) /(2 \eta)^{1 / 3}, \\
\mu & =(2 \eta)^{2 / 3}, \quad \eta \gg 0,|\rho-2 \eta|<2 \eta,
\end{aligned}
$$

and $\operatorname{Ai}(x)$ and $\operatorname{Bi}(x)$ are the Airy functions. For further details, see [3], [9], [21], [25].

$$
\begin{aligned}
F_{L}^{\prime}(\eta, \rho) \cdot G_{L}(\eta, \rho)-F_{L}(\eta, \rho) \cdot G_{L}^{\prime}(\eta, \rho) & =1 . \\
F_{L-1}(\eta, \rho) \cdot G_{L}(\eta, \rho)-F_{L}(\eta, \rho) \cdot G_{L-1}(\eta, \rho) & =L\left(L^{2}+\eta^{2}\right)^{-1 / 2} .
\end{aligned}
$$

In addition, the Leibniz rule for high order derivatives of products

$$
\begin{aligned}
(\alpha \cdot \beta)^{(k)}= & \alpha^{(k)} \cdot \beta+\left(\begin{array}{c}
k \\
1
\end{array}\right) \alpha^{(k-1)} \cdot \beta^{(1)}+\left(\begin{array}{l}
k \\
2
\end{array}\right) \alpha^{(k-2)} \cdot \beta^{(2)} \\
& +\cdots+\left(\begin{array}{c}
k \\
1
\end{array}\right) \alpha^{(1)} \cdot \beta^{(k-1)}+\alpha \cdot \beta^{(k)}
\end{aligned}
$$

is especially useful. Note that any one of the functions $\alpha \cdot \beta, \alpha$, or $\beta$ and its derivatives can be calculated in the proper sequence whenever the other two and their derivatives are known numerically.

3. Generation of Initial Values. We generally use two types of formulas. The first, applicable for reasonably small $\eta$ and $\rho$ values, consists of the integral representation (2.3) for $L=0$ and 1 ; the second, applicable for very large $\eta$, consists of the Airy function expansions (2.4) and (2.5).

Because the integral (2.3) is relatively difficult to evaluate with arbitrarily high precision, we recommend the procedure outlined below.

Each term in $F_{L}(\eta, \rho)$ or $G_{L}(\eta, \rho)$ contains an integral $\int_{0}^{\infty} \alpha(t) \beta(t) d t$ which is safely truncated at a suitably large $t$. The significant portion of this integral is evaluated to a specified degree of precision by a sum of integrals of the type $I(h)=$ $\int_{x}^{x+h} \alpha(t) \beta(t) d t . I(h)$, when expanded into the Taylor series

$$
I(h) \simeq \sum_{k=1}^{N} h^{k}[\alpha(x) \beta(x)]^{(k-1)} / k !,
$$

is easily computed from (2.8) when $\alpha, \beta$, and their derivatives are known numerically. For a given $N$ (the authors use $N=54$ with the CDC 3600 computer), a suitably large $h$ value is chosen, dependent on the magnitudes of the coefficients of the highest order terms used by the Taylor series, so that the truncation terms remain negligible.

If $T=1-\tanh ^{2} x=\operatorname{sech}^{2} x=4 z^{-1}$, then $z=e^{2 x}+e^{-2 x}+2, z^{(k)}=$ 
$2^{k}\left(e^{2 x}+(-1)^{k} e^{-2 x}\right)$ and $(z T)^{(k)}=0, k>0$. Thus, from (2.8), $T^{(k)}$ is obtained in terms of lower order derivatives of $T$ and $z$.

If $A=\rho \tanh x-2 \eta x, C=\cos A$ and $S=\sin A$, then $A^{(1)}=\rho T-2 \eta, A^{(1+k)}=$ $\rho T^{(k)}, S^{(1)}=C A^{(1)}$ and $C^{(1)}=-S A^{(1)} \cdot S^{(1+k)}$ and $C^{(1+k)}$ are available in the proper sequence from Leibniz's rule applied to $S^{(1)}$ and $C^{(1)}$.

If $v=-\rho x+2 \eta \arctan x$, the higher derivatives of $v$ are computed from (2.8) applied to the equation $\left(1+x^{2}\right)\left(v^{(1)}+\rho\right)=2 \eta$. When $B=e^{v}, B^{(1)}=B v^{(1)}$, and desired results follow naturally as before.

This technique obtained accurate values from which the more efficient approximation formulas were developed. It was also used to check numerical results obtained by other methods. It took approximately four minutes of CDC 3600 computer time to calculate $F_{L}(11,22)$ and $G_{L}(11,22)$ for $L=0$ and 1 to 22 decimal digit accuracy. The efficiency of this method improves for smaller $\eta$ and $\rho$ values.

The value $G_{0}^{\prime}(\eta, \rho)$ is determined by $G_{0}(\eta, \rho)$ and $G_{1}(\eta, \rho)$ according to Eq. (2.1).

4. Approximations for $G_{0}(\eta, \rho)$ and $G_{0}^{\prime}(\eta, \rho)$ Along Line Segments. By rearranging the Airy expansions (2.4) and (2.5) into continued fractions along the transition line $\rho=2 \eta$, we obtain

$$
G_{0}(\eta, 2 \eta) \simeq \lambda^{-1 / 12} \cdot G+\lambda^{7 / 12} \cdot H
$$

and

$$
G_{0}^{\prime}(\eta, 2 \eta) \simeq \lambda^{1 / 12} \cdot G^{\prime}+\lambda^{5 / 12} \cdot H^{\prime},
$$

where $\lambda=(2 \eta)^{-2}$ and $G, H, G^{\prime}$, and $H^{\prime}$ are of the form $G=G_{1} / 1-\lambda G_{2} / 1$ $-\lambda G_{3} / 1-\ldots$ with the coefficients listed in Table 1 . We can also derive these same expansions from the asymptotic series [9], [13]. Test calculations indicated that these continued fraction approximations are accurate over a much larger range than the corresponding Airy expansions from which they were derived.

Rational minimax approximations based on the Remes algorithm [6], [24] were ultimately obtained along the transition line for $1<\eta \leqq 15$ from the integration technique described in the preceding section, using intermediate Chebyshev polynomial approximations to speed the required computations. Coefficients for these approximations, with ranges $3.5<\eta \leqq 15,2<\eta \leqq 3.5$, and $1 \leqq \eta \leqq 2$, are given in Tables 2, 3, and 4, respectively.

In Table 5, we give coefficients for rational approximations along the line $\rho=1$ for $\eta<1$.

We also applied Eq. (1.1) to obtain numerical values for $G_{0}(\eta, 30)$ and $G_{0}^{\prime}(\eta, 30)$. In Tables 6 and 7, we have coefficients for rational approximations to these two functions for the intervals $15 \leqq \eta \leqq 18.5$ and $18.5<\eta \leqq 22$, and in Table 8 , we list coefficients for approximations to $\ln \left(G_{0}(\eta, 30)\right)$ and $\ln \left(-G_{0}^{\prime}(\eta, 30)\right)$ for $22<\eta \leqq 30$. These rational approximations at $\rho=30$ are given in terms of the Chebyshev polynomials $T_{0}(x)=1, T_{1}(x)=x$, and $T_{m}(x)=2 x \cdot T_{m-1}(x)-T_{m-2}(x)$, $m>1$, because the direct polynomial representations lead to cancellation errors.

Some relevant numerical values of these functions are provided in Table 9 for checking purposes. Test cases lead us to believe all digits in this table are significant, with possible roundoff errors in the last place. 
5. Calculation of $G_{L}(\eta, \rho)$. If the point $(\eta, \rho)$ is not on one of the line segments where our approximation formulas hold, then Eq. (1.1) determines both $G_{0}(\eta, \rho)$ and $G_{0}^{\prime}(\eta, \rho)$ at the desired point, assuming the initial values obtained from these rational approximations.

Since (1.1) implies

$$
\rho y^{(k+1)}=(2 \eta-\rho) y^{(k-1)}-(k-1)\left(y^{(k-2)}+y^{(k)}\right) \quad \text { with } k \geqq 1,
$$

the higher order derivatives are conveniently applied to Taylor series expansions for both $G_{0}(\eta, \rho)$ and $G_{0}^{\prime}(\eta, \rho)$. When the step size is varied and maximized according to magnitudes of high order terms in the series, the desired results are derived accurately in a relatively small number of steps. However, it may be necessary in certain regions to compute scaled derivatives $s^{k} \cdot y^{(k)} / k$ ! to keep intermediate values in the range of computer number systems.

Once $G_{0}(\eta, \rho)$ and $G_{0}^{\prime}(\eta, \rho)$ are known numerically, the consecutive $G_{L}(\eta, \rho)$ values for $L>0$ follow from Eqs. (2.1) and (2.2).

6. Checking of Results. The regular Coulomb functions were generally computed with the irregular functions for $L=0$ and 1 because common terms often appear in the mathematical formulas and because it is desirable to use the Wronskians (2.6) and (2.7) to check on obvious errors. Unfortunately, these Wronskians proved to be very poor checks on the validity of final results. The asymptotic, Airy, and Riccati methods described by Fröberg [9] show that the regular and irregular Coulomb wave functions are all based on common terms which are cancelled out completely in the Wronskians. In the asymptotic method, $\theta_{L}$ could be miscalculated by several orders of magnitude and the Wronskians would fail to show any inaccuracies.

The Airy function approximations (2.4) and (2.5) for $F_{0}(\eta, \rho), F_{0}^{\prime}(\eta, \rho), G_{0}(\eta, \rho)$ and $G_{0}^{\prime}(\eta, \rho)$, expanded to $\mu^{-36}$, were used to obtain initial values which were applied to the differential equation (1.1) to obtain these four functions at another point $\left(\eta, \rho_{1}\right)$. No significant error was detected by recomputing these four values from the Airy expansions at $\left(\eta, \rho_{1}\right)$. The initial values used in these tests were taken along the transition line $\rho=2 \eta$, where these Airy approximations are equivalent to the asymptotic series [1], [9], [13], [14], [15], [17]. For $11 \leqq \eta<15$, numerical results from the Airy expansions were found to be in very good agreement with those obtained from the integral expressions (2.3).

For $\eta \leqq 50, \rho>2 \eta+33$, numerical results from (4.1), (4.2), and (1.1) were compared to values obtained by the asymptotic method [9]. The function $\arg \Gamma(1+i \eta)$ required in the expression for $\theta_{L}$ was computed using a subroutine developed by Cody and Hillstrom [7].

For $\eta>50$, values obtained from two types of Riccati methods [9] were compared to results from (4.1), (4.2), and (1.1).

As formulas were verified, several CDC 3600 computer subroutines were designed to produce master values to at least 21 decimals. Later, a Fortran double precision subroutine for calculating $G_{L}(\eta, \rho)$ for $L=0,1, \cdots, n$ was designed for IBM 360 computers. This 360 subroutine, used on Argonne's 50-75 computer, calculated results for $L=0$ and 1 which were found to be accurate to at least $13 S$ for $\rho<2 \eta$ and to at least 13D when $\rho>2 \eta$ whenever $\rho>.2$. A test program using this sub- 
routine took less than 24 seconds to obtain all 800 values of $G_{0}(\eta, \rho)$ and all 800 values of $G_{0}^{\prime}(\eta, \rho)$ which appear in the U. S. National Bureau of Standards Handbook of Mathematical Functions [3].

7. Summary. The main purposes of this report are (1) to provide useful approximations to initial values of the commonly used differential equation, (2) to show how complicated integrals such as (2.3) can be numerically evaluated in a relatively efficient manner with high precision, and (3) to suggest methods which make it possible to obtain much more accurate results if they are ever required in the future.

8. Acknowledgments. The authors wish to express their appreciation to the referee for helpful comments, to $\dot{R}$. Buchal for a suggestion on checking results, to $\mathrm{K}$. Paciorek for determining limitations in some previously published formulas, and especially to W. J. Cody for many useful references, suggestions, and computer subroutines which made this project possible.

Applied Mathematics Division

Argonne National Laboratory

9700 South Cass Avenue

Argonne, Illinois 60439

1. M. Abramowitz, "Asymptotic expansions of Coulomb wave functions," Quart. Appl. Math., v. 7, 1949, pp. 75-84. MR 10, 454.

2. M. Abramowitz \& P. Rabinowitz, "Evaluation of Coulomb wave functions along the transition line," Phys. Rev., v. (2) 96, 1954, pp. 77-79. MR 16, 129.

3. M. Abramowitz \& I. A. Stegun (Editors), Handbook of Mathematical Functions with Formulas, Graphs and Mathematical Tables, Nat. Bur. Standards Appl. Math. Series, 55, U.S. Government Printing Office, Washington, D.C., 1964, pp. 446, 537-554. MR 29 \#4914.

4. L. C. Biedenharn, R. L. Gluckstern, M. H. Hull \& G. Breit, "Coulomb functions for large charges and small velocities," Phys. Rev., (2) v. 97, 1955, pp. 542-554. MR $16,697$.

5. J. Boersma, "Expansions for Coulomb wave functions," Math. Comp., v. 23, 1969, pp. 51-59. MR 38 \#6117.

6. W. J. CODY, W. Fraser \& J. F. HART, "Rational Chebyshev approximations using linear equations," Numer. Math., v. 12, 1968, pp. 242-251.

7. W. Cody \& K. Hillstrom, "Chebyshev approximations for the Coulomb phase shift," Math. Comp., v. 24, 1970, pp. 671-677. MR 42 \#8661.

8. A. R. Curtis, Coulomb Wave Functions, Royal Soc. Math. Tables, vol. 11, Cambridge Univ. Press, New York, 1964. MR 29 \#4915.

9. C. E. Fröberg, "Numerical treatment of Coulomb wave functions," Rev. Mod. Phy., v. 27, 1955, pp. 399-411. MR 17, 412.

10. W. Gautschi, "Algorithm 292-Regular Coulomb wave functions," Comm. ACM, v. 9, 1966, pp. 793-795.

11. W. GAUTSCHI, "Computational aspects of three-term recurrence relations," SIAM Rev., v. 9, 1967, pp. 24-82. MR 35 \#3927.

12. J. H. GuNN, "Algorithm 300-Coulomb wave functions," Comm. ACM, v. 10, 1967, pp. 244-245.

13. T. IsACson, "Asymptotic expansions of Coulomb wave functions on the transition line," Nordisk Tidskr. Informationsbehandling (BIT), v. 8, 1968, pp. 243-245. 280.

14. K. S. KölbIG, "Certification of algorithm 300," Comm. ACM, v. 12, 1969, pp. 279-

15. K. S. Kölbig, "Remark on algorithm 300," Comm. ACM, v. 12, 1969, p. 692.

16. Y. L. Luke, The Special Functions and Their Approximations. Vol. 1, Math. in Sci. and Engineering, vol. 53, Academic Press, New York, 1969, pp. 115-119, 134, 135, 212. MR 39 \#3039.

17. H. F. Lutz \& M. D. Karvelis, "Numerical calculation of Coulomb wave functions for repulsive Coulomb fields," Nuclear Phys., v. 43, 1963, pp. 31-44. 
18. A. S. Meligy \& E. M. El Gazzy, "On Coulomb wave functions," Proc. Cambridge Philos. Soc., v. 59, 1963, pp. 89-94. MR 27 \#357.

19. Tables of Coulomb Wave Functions. Vol. 1, Nat. Bur. Standards Appl. Math. Ser., no. 17, U.S. Government Printing Office, Washington, D.C., 1952. MR 13, 988.

20. R. L. Pexton, "Computer investigation of Coulomb wave functions," Math. Comp., v. 24,1970 , pp. $409-411$. MR $42 \# 7176$.

21. M. E. SherRY \& S. Fulda, "Calculation of gamma functions to high accuracy," $M T A C$, v. 13, 1959, pp. 314-315. MR 21 \#603.

22. I. A. Stegun \& M. Abramowitz, "Generation of Coulomb wave functions by means of recurrence relations," Phys. Rev., (2) v. 98, 1955, pp. 1851-1852. MR 16, 1155.

23. A. TuBIS, Table of Nonrelativistic Coulomb Wave Functions, LA-2150, Los Alamos, New Mexico, 1958 , pp. 1-277.

24. H. Werner, J. Stoer \& W. Bommas, "Rational Chebyshev approximation," Numer. $M a t h .$, v. 10,1967, pp. 289-306.

25. B. ZONDEK, "The values of $\Gamma(1 / 3)$ and $\Gamma(2 / 3)$ and their logarithms accurate to 28 decimals," $M T A C$, v. 9, 1955, pp. 24-25. MR 16, 861. 